\title{
Genetic Analysis of a Gene Regulating the Timing of Developmental Events in Dictyostelium discoideum
}

\author{
By K. ABE, Y. OKADA, M. WADA AND K. YANAGISAWA* \\ Institute of Biological Sciences, University of Tsukuba, Ibaraki 305, Japan
}

(Received 24 August 1982)

\begin{abstract}
In Dictyostelium discoideum, there exist genes which regulate the timing of developmental events. In one class of these rapidly developing (rde) mutants, the rate of development is accelerated, resulting in the formation of spores and stalk cells in about $2 / 3$ of the time required for the parent. Linkage analysis of one of the rde strains, HTY506, demonstrated that $r d e C 1850$, carried by the mutant is located on linkage group III. Therefore HTY 506 differs genetically from the strain FR17, the first rde mutant described which carries a mutation $r d e A 1$ on linkage group IV. While strain HTY506 has a pleiotropic phenotype, the altered characteristics of strain HTY506 appear to be caused by a single mutation, $r d e C 1850$.
\end{abstract}

\section{INTRODUCTION}

During development, organisms undergo a systematic series of morphological and biochemical changes. However, little is known about the mechanisms which regulate the timing of developmental events. In Dictyostelium discoideum, this timing has been shown to be affected by certain mutations (Sonneborn et al., 1963; Loomis, 1970; Atryzek, 1976; Kessin, 1977; Abe \& Yanagisawa, 1983). For example, in a strain FR17 (Sonneborn et al., 1963), all morphological and biochemical changes during development occur much earlier than in the wild-type parent without alteration in the normal order of these events. These mutants may be useful to study the temporal control of development.

Rapidly developing (rde) mutants have been isolated in several laboratories (Sonneborn et al., 1963; Atryzek, 1976; Kessin, 1977), and Kessin (1977) has reported that FR17 carries a recessive mutation, $r d e A 1$, located on linkage group IV.

To learn more about genetic control mechanisms of development, we have isolated a number of rde mutants and attempted to analyse these mutants genetically. We performed linkage analysis on one of the rde mutants, HTY506, and found that the mutation, $r d e C 1850$, carried by HTY506 is located on linkage group III. This suggests that there exist at least two mechanisms involved in regulating the timing of development of $D$. discoideum.

\section{METHODS}

Strains. Dictyostelium discoideum strains used were originally derived from the wild-type strain NC4. Genotypes of haploid strains are given in Table 1. The rapidly developing mutant strain HTY506 was derived from XP55 by $\gamma$-ray irradiation (Abe \& Yanagisawa, 1983). Strains NP2, XP55, XP95 and Bacillus subtilis 36.1 were kindly provided by Dr P. C. Newell (University of Oxford) and strain NP12 was a gift from Dr K. L. Williams (Max Planck Institute).

Growth and development. Amoebae were usually grown on nutrient agar plates in association with Klebsiella aerogenes at $22{ }^{\circ} \mathrm{C}$ (Sussman, 1966). For development of cells in liquid cultures, washed cells were suspended at a concentration of $1 \times 10^{7}$ cells $\mathrm{ml}^{-1}$ in $20 \mathrm{~mm}$-potassium phosphate buffer, $\mathrm{pH} 6.4$ and shaken on a rotary shaker at $22^{\circ} \mathrm{C}$ and 120 r.p.m.

Formation of heterozygous diploids. Heterozygous diploids were constructed according to the method of Newell $e t$ al. (1977). Diploids were usually recovered at a frequency of $1 \times 10^{-5}$. After two successive reclonings, diploid strains were identified by characteristic spore size, shape and genetic markers (Sussman \& Sussman, 1962, 1963). 
Table 1. Genotypes of haploid strains of Dictyostelium discoideum used

\begin{tabular}{|c|c|c|c|c|c|c|c|c|c|c|}
\hline \multirow[b]{2}{*}{ Strain } & \multicolumn{9}{|c|}{ Genotype* } & \multirow[b]{2}{*}{ Reference $\dagger$} \\
\hline & $c y c$ & whi & acr & axe & $t s q$ & bsg & $b w n$ & man & $c o b$ & \\
\hline XP55 & A5 & & & & & A5 & & & & a \\
\hline XP95 & & $\mathrm{Al}$ & $\mathrm{Al}$ & $\mathrm{Al}$ & $\mathrm{Al}$ & & Al & A2 & Al & a \\
\hline NP2 & & & & $\mathrm{Al}, \mathrm{Bl}$ & $\mathrm{Al}$ & & & & & $\mathrm{b}$ \\
\hline NP12 & & & $\mathrm{A} 2$ & $\mathrm{~A} 1, \mathrm{~B} 1$ & B3 & & & & & b \\
\hline
\end{tabular}

* Genotypic symbols are as follows: $c y c$, cycloheximide resistance; whi, white sorocarp; acr, acriflavin and methanol resistance; axe, axenic growth; $t s g$, temperature-sensitive growth; bsg, lack of growth on $B$. subtilis; bwn, brown pigment; man, deficiency in $\alpha$-mannosidase; $c o b$, cobalt resistance.

$\dagger \mathrm{a}$, Ross \& Newell (1979); b, Williams et al. (1974).

Haploidization. Haploid segregants were obtained from diploids plated clonally on nutrient agar plates containing the haploidizing agent, benlate at a concentration of $20-50 \mu \mathrm{g} \mathrm{m}^{-1}$ (Williams \& Barrand, 1978). The plating efficiency of diploids is about $30 \%$ at $30 \mu \mathrm{g}$ benlate $\mathrm{ml}^{-1}$. Haploid segregants appearing on benlate plates after 4-5 d were tested for their phenotypes using standard methods (Williams et al., 1974; Ratner \& Newell, 1978).

\section{RESULTS}

Rapidly developing mutants have been classified into two complementation groups, A and C (Abe \& Yanagisawa, 1983), the former corresponding to Kessin's rdeA mutants (Kessin, 1977), and the latter representing previously unreported groups of mutants. In this report, linkage analysis of the group C mutation in strain HTY506 is presented. Strain HTY506 was derived from XP55 by $\gamma$-ray irradiation resulting in approximately $10 \%$ suvival. HTY 506 formed heatresistant spore and stalk cells $16 \mathrm{~h}$ after the initiation of development, while the parental strain XP55 did not fully differentiate until $24 \mathrm{~h}$. This mutant also showed an abnormal final morphology, and was able to differentiate under conditions in which normal development was arrested without the addition of exogenous cAMP. For example, cells of HTY506 could form spore and stalk cells in liquid media $(60-70 \%$ of spores and $10-20 \%$ of stalk cells to total cells) and were able to form stalk cells without cell-to-cell contact. More detailed characteristics of group C mutants have been described (Abe et al., 1981; Abe \& Yanagisawa, 1983).

In order to assign $r d e C 1850$, carried by HTY 506 to a linkage group, the diploid strain DTY2 was constructed by parasexual fusion of HTY506 with a multiply marked tester strain, XP95. By plating spores of DTY2 on to benlate plates, we recovered several hundred haploid segregants whose phenotypes are shown in Table 2 . The most easily scored phenotype caused by $r d e C$ is aberrant final morphology. However, as the tester strain itself often shows abnormal fruiting structures, we also checked where necessary either, (1) the time required for spore formation or (2) the ability to differentiate into spore and stalk cells in liquid media.

The results demonstrated that the mutation $r d e C 1850$ segregated independently from the markers on linkage group I, VI and VII. Although a non-random segregation pattern was observed relative to linkage group II and IV, the presence of recombinant phenotypes demonstrated that the $r d e C$ locus is not on linkage group II and IV. In contrast, the marker $b s g A 5$ on linkage group III always co-segregated with $r d e C 1850$. Thus all the $\mathrm{RdeC}^{+}$segregants possess $t s g A$ and all the RdeC segregants are $b s g A$. Based on these results, we conclude that the mutation is located on linkage group III.

Two comments regarding these data must be made. First, the number of clones with the RdeC phenotype is several-fold higher than that of $\mathrm{RdeC}^{+}$. This is thought to be caused by a difference in growth rate on benlate plates between $\mathrm{RdeC}$ segregants and $\mathrm{RdeC}^{+}$segregants.

As shown in Fig. $1(a)$, while the growth rate of HTY506 was lower than the wild-type strain XP95, it still showed substantial growth on benlate plates, though strain XP95 barely grew on benlate. Only tiny colonies were produced even after $10 \mathrm{~d}$ growth. Furthermore, $\mathrm{RdeC}^{+}$ 


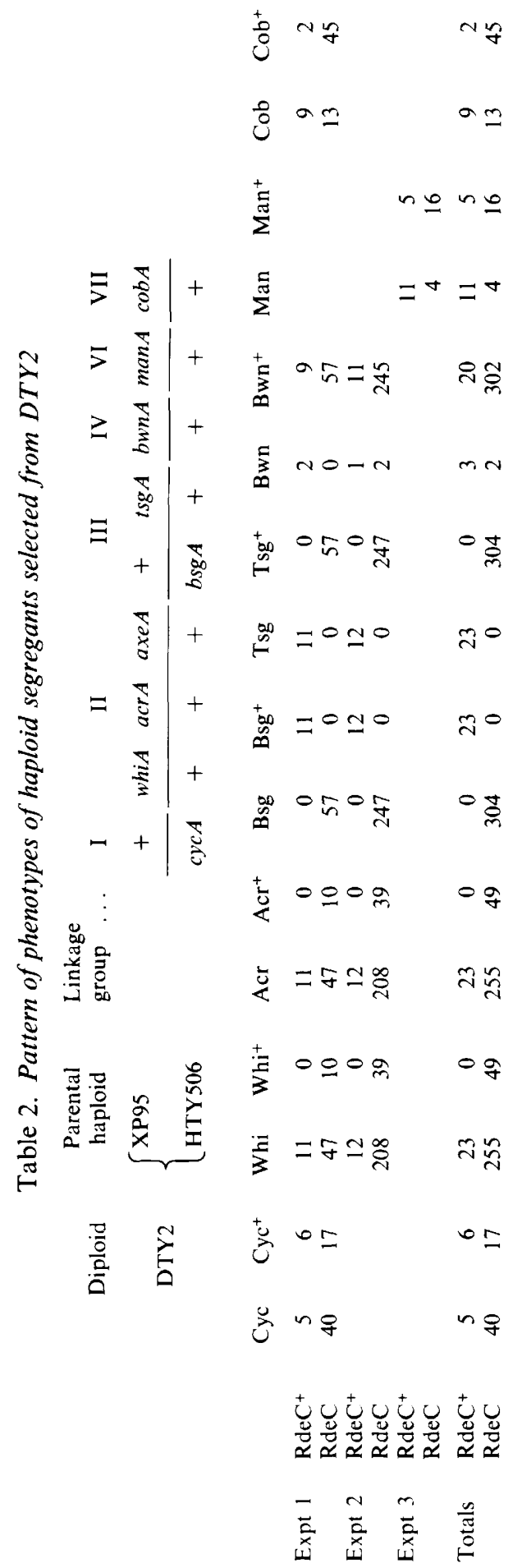




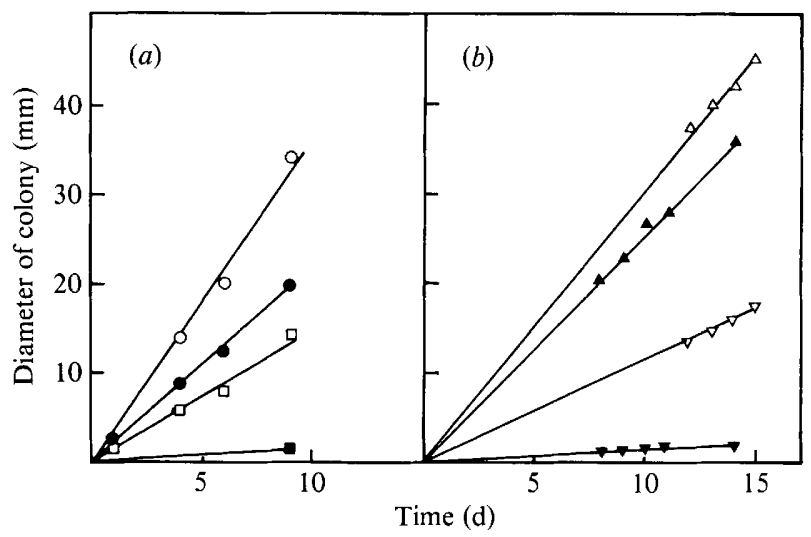

Fig. 1. Effect of benlate on growth rate of parental haploid strains and segregants. Cells of each strain were inoculated with toothpicks on to lawns of $K$. aerogenes growing on nutrient agar plates, with or without benlate $\left(30 \mu \mathrm{g} \mathrm{ml}^{-1}\right)$. Growth was measured by marking the boundaries of colonies at intervals. (a) Control, HTY506 (O), XP95 ( $\square$ ); benlate, HTY506 (๑), XP95 ( $\boldsymbol{\square})$. (b) Control, RdeC segregants $(\triangle), \operatorname{RdeC}^{+}$segregants $(\nabla)$; benlate, $\mathrm{RdeC}$ segregants $(\boldsymbol{\Lambda}), \mathrm{RdeC}^{+}$segregants $(\boldsymbol{\nabla})$.

segregants also grew poorly on benlate (Fig. $1 b$ ). Therefore, we assume that the relatively poor growth of $\mathrm{RdeC}^{+}$segregants on benlate results in a greater number of $\mathrm{RdeC}$ segregants than $\mathrm{RdeC}^{+}$

The second point concerns the apparent non-random segregation pattern observed relative to the markers on linkage group II and IV. For example, only three classes of haploid progeny involved with linkage group II were recovered. The $\mathrm{RdeC}^{+} \mathrm{AcrA}^{+} \mathrm{WhiA}^{+}$class of segregant from DTY2 was completely absent. Ross \& Newell (1979) reported similar results in the cross of XP55-derived mutants with XP95. They postulate that this is caused by unidentified mutations on chromosome II of XP95 and chromosome III of XP55 which individually are not harmful, but in combination are deleterious. Alternatively, since strains carrying an $a c r A$ mutation show cross-resistance to benlate (Williams \& Barrand, 1978), it is expected that the segregants carrying acr $A$ should grow rapidly on benlate. In addition, $\mathrm{RdeC}^{+}$segregants also grew poorly on benlate. Therefore, apparently $\mathrm{RdeC}^{+} \mathrm{AcrA}^{+} \mathrm{WhiA}^{+}$segregants did not appear on benlate. Nevertheless, the presence of the $\mathrm{RdeC}^{+} \mathrm{Acr} A$ WhiA and RdeC AcrA WhiA class of segregants clearly shows the absence of linkage of $r d e C$ with chromosome II.

In this cross, we also found that the appearance of segregants was highly skewed against the $b w n A$ marker. To re-examine the relationship of $r d e C$ locus to group IV, HTY 506 was crossed to NP12 (which carries a marker $t s g B 3$ mutation on linkage group IV) to form strain DTY 9 , from which haploid segregants were isolated using benlate. As may be seen in Table 3 , the $r$ deC 1850 mutation segregated independently from a marker $t \operatorname{sg} B 3$, while segregating with $b s g A 5$. These experiments confirmed earlier results that the $r d e C 1850$ mutation is located on linkage group III.

As mentioned above, $r d e C$ mutants were scored on the basis of their abnormal final morphology. In order to test the possibility that other characteristics of these mutants such as the capacity to differentiate in liquid media and rapid development are caused by the same mutation, we examined the characteristics of about 50 independently isolated haploid segregants from diploid DTY6 (Table 4). DTY6 was produced by the fusion of HTY506 (which carries $b s g A$ on linkage group III) with NP2 bearing $t s g A$ on group III. In all cases, segregants carrying chromosome III of HTY 506 formed spores and stalk cells by $18 \mathrm{~h}$ on a solid substratum. The fruiting structure had an aberrant morphology. All of the segregants could differentiate into spore and stalk cells in liquid culture. In contrast, segregants carrying chromosome III of NP2 produced normal fruiting-bodies with normal timing, and did not form spores and stalk cells in a shaken liquid suspension. These results suggest that the pleiotropic phenotype of group $\mathrm{C}$ mutants is caused by the same single mutation, and that if multiple mutations are involved, they must be linked. 
Table 3. Pattern of phenotypes of haploid segregants from DTY9

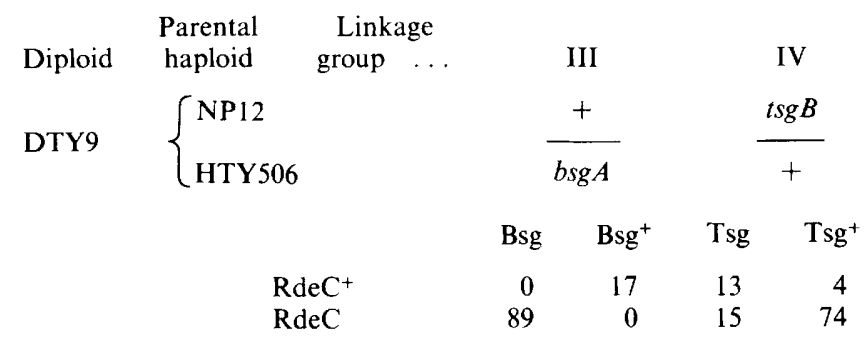

Table 4. Characteristics of haploid segregants from DTY6

$\begin{array}{lrrrrrr}\text { Normal } & \text { Aberrant } & \text { 18 h } & 24 \mathrm{~h} & + & - \\ \mathrm{Bsg} & 0 & 24 & 24 & 0 & 24 & 0 \\ \mathrm{Bsg}^{+} & 25 & 0 & 0 & 25 & 0 & 25 \\ \mathrm{Tsg}^{\text {Morphology }} & 25 & 0 & 0 & 25 & 0 & 25 \\ \text { Tsg }^{+} & 0 & 24 & 24 & 0 & 24 & 0\end{array}$

* + indicates that cells differentiate into considerable number of spores (about $60-70 \%$ of total cells) in a liquid suspension culture by $20 \mathrm{~h}$ after the initiation of development; - indicates no spores appeared in liquid culture.

\section{DISCUSSION}

The pleiotropic nature of the group $\mathrm{C}$ mutant phenotype could indicate multiple mutations occurring within the same linkage group. However, the characteristics of group $\mathrm{C}$ mutants always co-segregated and independent group $\mathrm{C}$ mutants could be isolated frequently, even after a relatively mild mutagenic treatment (about $10 \%$ survival). For example, of the 16 rde mutants isolated, 10 were group $\mathrm{C}$ mutants, while only two were from group $\mathrm{A}$, which is believed to carry a single mutation (Kessin, 1977). These results suggest that the pleiotropic phenotype of group $\mathrm{C}$ mutants is caused by a single mutation.

Several research groups have isolated mutants showing phenotypes similar to our rde mutants (Sonneborn et al., 1963; Atryzek, 1976; Kessin, 1977). The assignment of rdeC1850 to linkage group III confirmed our previous conclusion that group $\mathrm{C}$ mutants differ genetically from group A mutants carrying $r d e A$ on linkage group IV. While another group of rde mutants, group B (i.e. $r d e B$ ), has been described (Kessin, 1977), these strains have been lost, and were therefore not available for inclusion in our genetic analysis. However, several non-genetic characteristics suggest that our group $\mathrm{C}$ mutants differ from these group B mutants, as well as group A mutants. In group $\mathrm{A}$ and group $\mathrm{B}$ mutants, cell aggregation occurs several hours earlier than in their parental strains, while group $\mathrm{C}$ mutants aggregate with normal timing. In group $\mathrm{C}$ mutants, it is mainly the time required for spore and stalk cell differentiation following the completion of aggregation that is reduced (Abe \& Yanagisawa, 1983). This suggests that there are at least two mechanisms involved in regulating the timing of development of $D$. discoideum.

The phenotypes of sporogenous mutants, described by Gross's group (Town et al., 1976; Gross et al., 1981), share some characteristics in common with our rde mutants, e.g. abnormal final morphology and differentiation under unusual conditions. An analysis between the sporogenous mutants and our rde mutants would be interesting.

To date, the genes affected by the rde mutations are not known, however, cAMP metabolism is significantly altered in both group A and group C mutants (Coukell \& Chan, 1980; Abe \& Yanagisawa, 1983). Characteristics similar to those seen in the rde mutants, can be induced by the addition of cAMP to wild-type cells (Gerisch et al., 1975; Town et al., 1976; Feit et al., 1978; Nestle \& Sussman, 1972). Hence, the phenotypes of rde mutants may be caused by their altered cAMP metabolism. 
Further genetic and biochemical studies on rde mutants will provide clues to identity of the genes which regulate the timing of development.

\section{REFERENCES}

ABE, K. \& Yanagisawa, K. (1983). A new class of rapidly developing mutants in Dictyostelium discoideum: implications for cyclic AMP metabolism and cell differentiation. Developmental Biology 95, 200210 .

Abe, K., Saga, Y., Okada, H. \& Yanagisawa, K. (1981). Cell differentiation of Dictyostelium discoideum mutant cells in a shaken suspension culture and the effect of cyclic AMP. Journal of Cell Science 51, $131-142$.

ATRYZEK, V. (1976). Alteration in timing of cell differentiation resulting from cell interactions during development of the cellular slime mold Dictyostelium discoideum. Developmental Biology 50, 489-501.

Coukell, M. B. \& Chan, F. K. (1980). The precocious appearance and activation of an adenylate cyclase in a rapid developing mutant of Dictyostelium discoideum. FEBS Letters 110, 39-42.

Feit, I. N., Fournier, G. A., Needleman, R. D. \& UNDER WOOD, M. Z. (1978). Induction of stalk and spore cell differentiation by cyclic AMP in slugs of Dictyostelium discoideum. Science 200, 439-441.

Gerisch, G., Fromm, H., Huesgen, A. \& Wick, U. (1975). Control of cell-contact sites by cyclic AMP pulses in differentiating Dictyostelium cells. Nature, London 255, 547-549.

Gross, J. D., Town, C. D., Brookman, J. J., Jermyn, K. A., Peacey, M. J. \& Kay, R. R. (1981). Cell patterning in Dictyostelium. Philosophical Transactions of the Royal Society B295, 497-508.

Kessin, R. H. (1977). Mutations causing rapid development of Dictyostelium discoideum. Cell 10, 703-708.

Loomis, W. F. (1970). Temporal control of differentiation in the slime mold, Dictyostelium discoideum. Experimental Cell Research 60, 285-289.

Nestle, M. \& Sussman, M. (1972). The effect of cyclic AMP on morphogenesis and enzyme accumulation in Dictyostelium discoideum. Developmental Biology 28, 545-554.

Newell, P. C., Henderson, R. F., Mosses, D. \&
RATNER, D. I. (1977). Sensitivity to Bacillus subtilis: a novel system for selection of heterozygous diploids of Dictyostelium discoideum. Journal of General Microbiology 100, 207-211.

Ratner, D. I. \& Newell, P. C. (1978). Linkage analysis in Dictyostelium discoideum using multiply marked tester strain: establishment of linkage group VII and the reassessment of earlier linkage data. Journal of General Microbiology 109, 225-236.

Ross, F. M. \& Newell, P. C. (1979). Genetics of aggregation pattern mutations in the cellular slime mould Dictyostelium discoideum. Journal of General Microbiology 115, 289-300.

Sonneborn, D. R., White, G. J. \& Sussman, M. (1963). Mutation affecting both rate and pattern of morphogenesis in Dictyostelium discoideum. Developmental Biology 7, 79-93.

SuSSMAN, M. (1966). Biochemical and genetic methods in the study of cellular slime mold development. Methods in Cell Physiology 2, 397-410.

Sussman, M. \& Sussman, R. (1962). Ploidal inheritance in Dictyostelium discoideum: stable haploid, stable diploid and metastable strains. Journal of General Microbiology 28, 417-429.

Sussman, R. \& Sussman, M. (1963). Ploidal inheritance in the slime mould Dictyostelium discoideum: haploidization and genetic segregation of diploid strains. Journal of General Microbiology 30, 349-355.

Town, C. D., Gross, J. D. \& KAY, R. R. (1976). Cell differentiation without morphogenesis in Dictyostelium discoideum. Nature, London 262, 717-719.

Williams, K. L. \& BarRand, P. (1978). Parasexual genetics in the cellular slime mould Dictyostelium discoideum: haploidization of diploid strains using benlate. FEMS Microbiology Letters 4, 155-159.

Williams, K. L., Kessin, R. H. \& Newell, P. C. (1974). Parasexual genetics in Dictyostelium discoideum: mitotic analysis of acriflavin resistance and growth in axenic medium. Journal of General Microbiology 84, 59-69. 